\title{
JEAN SCHLUMBERGER VU À TRAVERS LA CORRESPONDANCE DE PROUST: DES MONDANITÉS LITTÉRAIRES À LA CRÉATION ROMANESQUE
}

\begin{abstract}
Fraisse Luc, Jean Schlumberger vu à travers la correspondance de Proust: des mondanités littéraires à la création romanesque [Jean Schlumberger in Proust's Correspondence. From literary salons to novel writing]. Studia Romanica Posnaniensia, Adam Mickiewicz University Press, Poznań, vol. XXXI: 2004, pp. 23-34. ISBN 83-232-1353-4, ISSN 0137-2475.
\end{abstract}

The name of Jean Schlumberger, the founding member of «Nouvelle Revue Française», which appears at times in Marcel Proust's letters, illustrates well the possibilities that the writer's correspondence opens up for a researcher, who wants to recreate his line of reasoning and creative process. Jean Schlumberger and his cousin Gustave at first seem to appear in literary circles known to Proust as quitc negative figures, whose views on almost every subject he did not share. Gradually, however, the author of $L a$ Recherche revises his stance and as a result of his contact with Schlumberger he rewrites different fragments of his novel cycle, especially one episodc of Sodome et Gomorrhe and some theoretical passage of Temps retrouvé. Only the reference to the letters enables us to perceive the mystcrious way leading from sociological surface to the depth of creative process.

Les apparitions de Jean Schlumberger dans la correspondance de Proust illustrent pleinement la portée polyvalente de ces lettres, à la faveur desquelles les mondanités littéraires apparemment les plus superficielles (et qui pour cette raison irritent un certain nombre de lecteurs) se trouvent nounir par des voies secrètes la création romanesque la plus complexe qui soit, celle de la Recherche du temps perdu. Jean Schlumberger, arrière-petit-fils de Guizot, membre fondateur de la «Nouvelle Revue Française», et cousin de Gustave Schlumberger, connaît avec Proust des relations essentiellement évolutives qui offrent le double intérêt de faire apercevoir comment une certaine forme de sociabilité peut aboutir à l'invention, mais aussi par ce moyen comment une rencontre placée a priori sous les auspices les plus négatifs devient peu à peu, non seulement enrichissante, mais source directe de création: ce n'est pas sans étonnement que l'on découvrira en effet que Jean Schlumberger a inspiré au romancier de la Recherche en partie la structure d'un 
épisode de Sodome et Gomorrhe et, bien plus directement, un passage célèbre et dogmatique du Temps retrouvé. C'est l'histoire de ce renversement, qui va de la superficie sociale aux profondeurs de la création, que l'on voudrait retracer ici; chemin faisant s'éclairera en outre d'un jour oblique la signification d'une correspondance d'écrivain - puisque tel est ici le support de l'enquête - dans l'élaboration d'une cuvre.

C'est bien dans la mouvance de la «Nouvelle Revue Française» que Proust rencontre, donc assez tard au cours de son évolition littéraire personnelle, Jean Schlumberger. Celui-ci a visiblement servi d'intermédiaire, en 1913, entre celui qui publiera en novembre $D u$ côté de chez Swann et Jacques Copeau, dans la souscription de trois actions que le romancier contracte en faveur du théâtre du VieuxColombier, qui sera inauguré le 23 octobre $^{1}$. C'est donc un Proust à la fois excessivement prévenant et compliqué à outrance que découvre Schlumberger, notamment lorsqu'il reçoit ce billet, le 14 juin 1913:

Je ne vous retourne pas la feuille signée, parce que je crois que vous me l'avez envoyée par erreur. J'ai reçu avant-hier de M. Copeau une feuille analogue, je l'ai signée et envoyée au Limon. II me semble que celle-ci fait double emploi, et que si je la signais aussi pour 3 actions, j'aurais l'air de m'engager à en prendre non plus 3, mais 6 . Il est très possible que je comprenne mal. Si j'ai raison et si vous m'avez adressé cette feuille seulement parce que vous ignoricz que je venais d'en recevoir une de $\mathbf{M}$. Copeau, ne prenez pas la peine de me répondre. Votre silence signifie que je n'ai rien à faire. Dans le cas contraire, veuillez me récrire ${ }^{2}$.

On touche ici aux aléas d'une relation épistolaire, qui diffère et entrecroise les questions et les réponses, lesquelles s'accumulent chez un écrivain à la fois reclus et attentif à l'activité littéraire parisienne. Car sont en jeu ici les relations curieuses entre Proust et le théâtre ainsi que l'actualité littéraire, le reclus du boulevard Haussmann se trouvant à la fois coupé et pourtant tout proche du monde extérieur parisien. L'inauguration du théâtre du Vieux-Colombier l'intéresse, comme tout ce qui touche à la «Nouvelle Revue Française», dont il a perçu très tôt la valeur et l'avenir littéraires, mais aussi les réticences à l'égard de son œuvre: Gallimard refuse, on le sait, de publier $D u$ côté de chez Swann, et Gide et certains de ses collaborateurs se méfient de l'auteur de la Recherche.

Ce sont donc au départ des relations de méfiance mutuelle qui s'instaurent, entre Proust et Schlumberger. On le voit à un détail significatif. Au tout début de 1914, au moment où la critique rend compte de Swann qui vient de paraitre, les relations avec la «Revue» de Gide reprennent. Le romancier indique notamment à Jacques Copeau à quels membres de l'équipe de rédaction il a fait parvenir son

${ }^{1}$ Correspondance de Marcel Proust, présentée, établie et annotée par Philip Kolb, Plon, 21 vol., Paris 1970-1993; ici t. XII, p. 181, note 9.

${ }^{2}$ Ibidem, p. 200. 
volume, car il est très attentif à l'opinion de ce groupe intellectuel, à deux exceptions près qu'il faut relever:

Oui j'ai envoyé le livre à $M$. Suarès, du reste moi qui ai fait peu d'envois de ce livre, une bonne moitié a été pour les principaux rédacteurs de la N.R.F. (par qui cela m'intéressait d'être lu). Je n'avais excepté, pour des raisons différentes, que M. Ghéon et M. Schlumberger. Mais si mes exemplaires sont parvenus, M. Claudel, M. Gide, M. Thibaudet, M. Fargue, M. Gallimard, ont dû recevoir ce livre. Et vous, avant tous ${ }^{3}$.

La réticence marquée à l'égard de Schlumberger s'explique de deux manières. Le critique partage d'abord avec Henri Ghéon ce qui semble à Proust une méconnaissance de ce qu'est la profondeur littéraire.

Nous l'apprenons par une grande lettre de réfutation, écrite le 2 janvier 1914 à Henri Ghéon qui vient d'éreinter ironiquement $D u$ côté de chez. Swann dans la N.R.F. parue la veille. Ghéon voit en cet écrivain le contraire d'un romancier, soit un auteur qui fait directement passer dans son wuvre toutes les caractéristiques de sa vie et de sa personnalité, sans le moindre effort de recréation. L'écrivain argumente dès lors:

moi qui mène la vie d'un malade, pas une fois je n'ai écrit la psychologie, le 'roman' du malade. (Jamais je n'aurais écrit la page de $\mathrm{M}$. Werth à laquelle un de vos collaborateurs trouve de l' 'accent' (?). Si je parle de maladie dans les volumes suivants, c'est une maladic inventéc pour les besoins psychologiques de l'œuvre".

C'est Louis de Robert qui avait publié quant à lui, en mars 1911, chez Fasquelle, Le Roman du malade; mais c'est Jean Schlumberger qui, rendant compte dans ce même numéro de la N.R.F. de La Maison blanche de Léon Werth, introduisait la citation d'un passage du livre sur la maladie par cette réflexion: «Charles-Louis Philippe eût aimé cet accent» ${ }^{6}$. On sait que pour le romancier de la Recherche, l'accent d'un artiste, celui de la phrase de Bergotte, et bien plus celui que fera entendre le septuor de Vinteuil dans La Prisonnière?, traduit l'essence la plus mystérieuse et l'originalité la plus profonde qu'apporte chaque artiste au monde: aussi la notion lui semble-t-elle galvaudée, au profit d'un livre très secondaire. Un lien logique relie en fait à ses yeux l'éloge de Schlumberger et la critique ironique de Ghéon, l'un et l'autre louant et stigmatisant symétriquement à

${ }^{3}$ Idem, t. XIII, p. 21.

${ }^{4}$ Ibidem, p. 25.

${ }^{5}$ Proust en reprend aujourd'hui le titre comme repoussoir, ce qui n'empêche qu'il ait apprécié ce livre et l'ait tenu en haute estime, comme il l'écrivait à son auteur au moment de la parution: «pour ceux qui, comme moi, croient que la littérature est la dernière expression de la vie, si la maladie vous a aidé à écrire ce livre-là, ils penseront que vous avez dû accueillir sans colère la collaboratrice inspirée» (idem, t. X, p. 271).

${ }^{6}$ Idcm, t. XIII, p. 29, note 16.

${ }^{7}$ ̀ la recherche $d u$ temps perdu, édition réalisée sous la direction de Jean-Yves Tadié, Gallimard, «Bibliothèque de la Pléiade», 4 vol., Paris 1987-1989; ici t. III, pp. 760 sqq. 
contretemps des œuvres contemporaines: c'est exactement ce que Proust reprochait à Sainte-Beuve, méconnaissant les grands créateurs de son temps, tout occupé qu'il était à encenser des écrivains de troisième ordre ${ }^{8}$.

À cette déception intellectuelle s'ajoute que Schlumberger porte le nom de l'une des rares personnes que Proust, en dépit de sa générosité sans limites, ait authentiquement et âprement détestée, à savoir Gustave Schlumberger. Cette haine apparait, à l'horizon de Ja relation de Proust à Jean Schlumberger à ses débuts: ce sont ces prémisses que l'avenir parviendra à redresser peu à peu. Il faut donc dire ici quelques mots de l'historien de la civilisation byzantine, car les lettres de Proust à son sujet pourraient aisément s'insérer dans l'ouvrage de Sainte-Beuve qui s'intitule Mes Poisons. Nommé quant à lui dans la correspondance très tôt, soit dès $1894^{9}$, Gustave Schlumberger témoignera lui-même, dans ses Souvenirs parus en 1934, de ce qu'il n'a jamais compris Proust: «Sur un tabouret, aux pieds de Mme Geneviève Straus, on voyait constamment le bizarre Marcel Proust, encore adolescent, qui, depuis, a écrit des livres admirés des uns, incompréhensibles pour les autres, dont je suis» ${ }^{10}$.

Gustave Schlumberger représente pour Proust l'écrivain bourgeois reçu chez les aristocrates, un Swann mais sans finesse, et qu'il regarde en sociologue: à l'époque où il se documente pour écrire Jean Santeuil et étoffer le personnage du duc de Réveillon qui deviendra le duc de Guermantes dans la Recherche, il voudrait savoir par quel geste (tendre la main gauche?) un duc pourrait marquer un certain mépris à tel écrivain de ses hôtes, - disons un Gustave Schlumberger ${ }^{11}$. Mais la haine de Proust date du jour où l'historien, au moment de l'affaire Dreyfus, déserte le salon de Mme Straus, avec Jules Lemaitre et Forain, après le soir où Joseph Reinach entreprit de prouver que le vrai coupable n'était pas Alfred Dreyfus mais Estherhazy $^{12}$. Ce n'est pas tant ici le partisan de Dreyfus et l'admirateur de Reinach qui se révoltent en Proust, que l'adulateur de Mme Straus, aux pieds de qui en effet il restera (même au fond de sa réclusion ultérieure) durant toute sa vie. Par fidélité à

${ }^{8}$ Contre Sainte-Beuve, publié avec Pastiches et mélanges et Essais et articles par Pierre Clarac et Yves Sandre, Gallimard, «Bibliothèque de la Plćiade», Paris 1971, p. 223. Après avoir rappelé les réticences de Sainte-Beuve à l'égard de Balzac et Stendhal, il ajoute: «il est bien clair que, si tous les ouvrages du XIX ${ }^{c}$ siècle avaient brûlé sauf les Lundis, et que ce soit dans les Lundis que nous dussions nous faire une idée des rangs des écrivains du XIX siècle, Stendhal nous apparaîtrait inféricur à Charles de Bernard, à Vinct, à Molé, à Mme de Verdelin, à Ramond, à Sénac de Mcilhan, à Vicq d'Azyr, à combien d'autres, et assez indistinct, à vrai dire, entre d'Alton Shée et Jacquemont». Transposons: si l'on devait ne se faire une idée de la littérature du début du $\mathrm{XX}^{c}$ siècle que d'après les chroniques d'Henri Ghéon et Jean Schlumberger dans la «Nouvelle Revue Française», un Marcel Proust y apparaîtrait assez indistinct au milieu d'une pléiade de Léon Werth.

${ }^{9}$ Correspondance, t. I, p. 329.

${ }^{10}$ Gustave Schlumberger, Mes souvenirs, 1844-1928, Plon, Paris 1934, t. I, p. 305; voir Correspondance, t. I, p. 330 , note 7 .

${ }^{11}$ Correspondance, t. II, p. 215.

${ }^{12}$ Idem, t. XIV, p. 37, note 5: Proust rappelle ici l'événement à Joseph Reinach lui-même. 
son amie de toujours et pour toujours, il veut écrire au «Figaro» en 1908 pour torpiller la candidature du traître à l'Académie ${ }^{13}$, pousse implicitement Robert de Montesquiou au même moment ${ }^{14}$, et explicitement Édouard Rod l'année suivante ${ }^{15}$, à se porter candidats contre lui, et ne se montre satisfait que lorsqu'à l'entrée d'une soirée mondaine, il a pu lui infliger un affront public ${ }^{16}$.

Une extraordinaire décharge de haine se dégage de toutes ces lettres, si rares en effet sous la plume de Proust épistolier. Très tôt cependant et plus encore avec le temps, les charges contre Gustave Schlumberger, qui resteront un thème épistolaire intermittent, connaissent une élaboration littéraire qui évolue en parallèle avec la peinture de la société des Guermantes dans la Recherche, et nous découvre une facette de l'écrivain qui ailleurs trouve peu à se faire jour, le mémorialiste de son temps. Le thème dominant est l'apparition du personnage en milieu mondain, sous l'aspect peu avantagé d'un animal lourd. Devant les échecs de ses candidatures à l'Académie, Schlumberger devient, dès 1909 , un "pachyderme désabusé» ${ }^{17}$. Mais c'est durant les deux dernières années de la vie de Proust que l'auteur du Côté de Guermantes (publié de fait en 1920 et 1921) donne le plus abondamment libre cours à sa verve de portraitiste. C'est en mars 1921 que le romancier découvre une parenté entre Gustave autrefois connu et Jean qu'il approche un peu aujourd'hui, et auquel il confie:

Hélas je me souviens qu'au temps lointain où je voyais plusieurs fois par semaine un homme qui me détestait mais me montrait la plus grande affabilité, M. Gustave Schlumberger, il me parlait quelquefois d'une lointaine famille protestante. Quand j'ai commencé à vous lire, pas un instant je n'ai pu établir un lien quelconque (c'est la première fois que j’y pense [...]) entre vous et cet homme dont les notices dans les Débats sur une étoffe byzantine ou sur une croisade m' amuseraient si je ne l'avais pas connu ${ }^{18}$.

Il est curieux de noter que Proust, à cette date, récrit assez minutieusement ce qu'il a déjà publié dans la Recherche, à propos de Vinteuil dans Du côté de chez Swann édité en 1913, et de Bergotte dans À l'ombre des jeunes filles en fleurs paru en 1919. Car la théorie sous-jacente contre Sainte-Beuve est à nouveau fortement présente ici, adaptée tour à tour aux deux Schlumberger ou au seul Gustave. Comme Bergotte cesse d'être le doux chantre, dès l'instant qu'ayant lu ses livres, on aperçoit l'homme ${ }^{19}$, les études savantes de l'historien voient leur charme annihilé par le souvenir de l'homme en société. Plus subtilement, l'impossibilité qu'il y eut à supposer pour Proust que l'écrivain Jean et le pachyderme Gustave

\footnotetext{
${ }^{13}$ Idem, t. VIII, pp. 133-134 et 139-141.

${ }^{14}$ Idem, t. IX, pp. 248-249.

${ }^{15}$ Ibidem, p. 29.

${ }^{16}$ Idem, t. VIII, pp. 144-145.

${ }^{17}$ Idem, t. IX, p. 94.

${ }^{18}$ Idem, t. XX, pp. 158-159.

${ }^{19}$ Recherche, t. I, pp. 537 sqq.
} 
pussent être apparentés, reproduit l'épisode d' «Un amour de Swann» où celui-ci, entendant nommer Vinteuil comme l'auteur de la sublime sonate, ne peut croire qu'il s'agisse du même être que l'on appelait à Combray «cette vieille bête de Vinteuil ${ }^{20}$ : les deux Schlumberger apparaissent comme un dédoublement rétrospectif de la double personnalité du musicien de la Recherche, et de tout artiste aux yeux de Proust.

Le portrait charge peut alors commencer, même s'il y a lieu de s'étonner que l'épistolier l'adresse à Jean Schlumberger lui-même, dont il perçoit la parenté (sans d'ailleurs, on le verra, exactement l'avoir précisée) avec sa victime:

il affectait une horreur exaspérée de tous les protestants, et était d'une frivolité à donner l'impression quand on le quittait que Mme Edmond de Pourtalès était un bas-bleu. Avant même que l'affaire Dreyfus ne nous divisât, nous n'avions absolument rien à nous dire, ce qui, quand nous allions d'une soirce à une autre, avait quelque chose de lugubre. L'affaire Dreyfus etant un motif de discussion eût pu l'être de conversations. Mais il (M. Gustave Schlumberger) commençait par déclarer: 'je ne veux rien savoir, ne rien lire, je ne lis rien'. Il vous entendait 'sur l'affaire Dreyfus', mais je crois bien qu'il prenait le tout pour la partie et qu'il avait commencé depuis longtemps à ne rien lire. Il jouait: un certain bridge porte son nom. Mais il ne lisait pas. Peut-être maintenant depuis tant et tant d'années que je nc l'ai vu, a-t-il changé et se défend-il seulement de lire les ouvrages qu'il n'aime pas. Je sais en cffet qu'il a déclaré dernièrement qu'il aimerait mieux être condamné à mort et exécuté, plutôt que de lire un de mes livres. Je ne puis admirer exagérément cette bravoure, puisque personne ne s'aviserait jamais de lui imposer une telle alternative ${ }^{21}$.

La fermeture à l'égard de l'affaire Dreyfus est mise, on le voit, en équivalence avec celle à l'égard de la littérature: ne rien vouloir savoir sur Alfred Dreyfus conduit à ne rien vouloir lire de Marcel Proust.

Mis en verve par la rédaction de cette lettre, Proust reviendra à la charge durant l'année 1922. C'est auprès de Robert de Flers qu'en juillet, il distingue à présent soigneusement, nous verrons dans un instant pourquoi, Jean Schlumberger de «son imbécile d'oncle que j'ai vu encore l'autre jour chez Mme de Mun [...] s'ébrouer dans l'antichambre, friser ses moustaches de Vercingétorix en toc, se précipiter sur Mme de Ganay à qui on avait passé une muselière de rubis pour qu'elle ne pût pas ronger les ongles du pauvre M. Gérard de Ganay [...]. Puis le faux Schlumberger (je veux dire Gustave, le vrai étant Jean) a quitté cette avide Béhague pour se réfugier sous l'aile de la comtesse Murat, laquelle lui a tellement craché à la figure pour lui expliquer que l'élection de Maurras [à l'Académie] s'imposait, qu'il est parti ruisselant comme un hippopotame» ${ }^{22}$. L'anecdote se poursuit cependant auprès de Jean Schlumberger lui-même, à qui Proust résume le récit précédent, avant de poursuivre ainsi:

\footnotetext{
${ }^{20}$ Ibidem, pp. 210-211.

${ }^{21}$ Correspondance, t. XX, p. 159.

${ }^{22}$ Idem, t. XXI, pp. 353-354.
} 
Quand la musique fut finie, votre oncle sentant la soirée finic, se leva, ronchonna contre le 'monde' et les soirées 'son plus grand supplice', déclara-t-il, ce qui excita l'admiration de Mme de Ganay elle-même devant l'abnégation quotidienne et depuis tant d'années d'un mondain par mortification. Malheureusement causant pendant la musique [...], le pauvre Gustave Schlumber [sic] n'avait pas regardé le programme et ignorait que la première partie seulement de la soirée avait eu lieu. Il descendait déjà, furibond, trompé, et exhalant ses goûts de solitaire, les premières marches de l'escalier, quand la seconde partie du programme commença. Aussitôt avec une prestesse, méritoire chez ce sauvage ermite, il remonta les marches descendues et courut se blottir entre deux duchesses ${ }^{23}$.

Livrant entre-temps à une autre destinataire un pastiche de soirée mondaine intitulé «Conversation bête entendue chez une femme remarquable», l'épistolier imagine ce dialogue qui résume tout: «- Quel est ce buffle qui entre en s'ébrouant et va chercher un refuge sous l'aile de Thérèse Murat? - C'est Gustave Schlombec» 24 .

Le romancier humoriste est ici au travail, on le voit, un romancier légèrement différent de celui de la Recherche quoique lui ressemblant beaucoup, délivré en effet des visées dogmatiques du cycle romanesque, et pouvant dès lors par exception se livrer à la pure verve du brillant mémorialiste. L'attitude de Schlumberger durant le concert n'est pas sans rappeler, moins à cette date celle des aristocrates durant le concert donné chez la marquise de Saint-Euverte dans «Un amour de Swann» ${ }^{25}$ que celle qu'adopteront bientôt les invités de Mme Verdurin, dans La Prisonnière alors encore à paraître, quand on exécute le septuor de Vinteuil $^{26}$. Mais une cristallisation plus importante est en train de se jouer dans ces lettres, contemporaines de l'achèvement de Sodome et Gomorrhe $I I$, publié le 29 avril 1922: un lien secret unit en fait ici le nom de Schlumberger et, dans le roman, l'univers des Legrandin-Cambremer.

Reprenons les éléments dans l'ordre. En mars 1921, Proust avoue à Jean Schlumberger avoir cru sa famille propriétaire d'un château, Le Val-Richer, près de Cambremer en Calvados, que possèdent en effet MM. et Mmes P. et C. de Schlumberger ${ }^{27}$. Or, on sait que près de Balbec, dans Sodome et Gomorrhe, les Verdurin ont loué une propriété, la Raspelière, que possède la famille Cambremer. La jeune Mme de Cambremer, dès «Un amour de Swann», s'est piquée, non sans snobisme, de savoir authentiquement goûter la musique, contrairement aux aristocrates généralement ignorants (tel apparemment Gustave Schlumberger) qui l'entourent ${ }^{28}$, ce que confirment ses conversations avec le héros de la Recherche dans Sodome et Gomorrhe $e^{29}$. Sa belle-mère, Mme de Cambremer douairière, an-

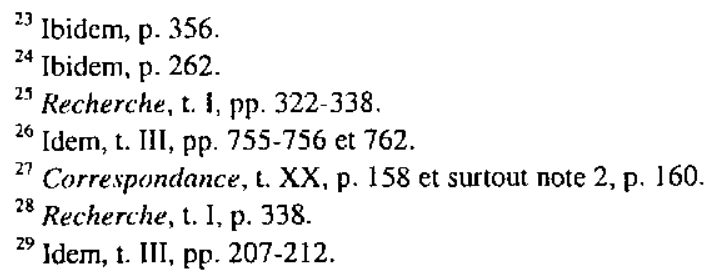


cienne élève de Chopin, est cependant une authentique musicienne; mais son goût en art se manifeste par un phénomène étrange, dont Proust romancier se montre très fier dans ses lettres, à savoir une salivation intempestive suscitée par l'enthousiasme:

Chaque fois qu'elle parlait esthétique ses glandes salivaires, comme celles de certains animaux au moment du rut, entraient dans une phase d'hypersécrétion telle que la bouche édentée de la vieille dame laissait passer au coin des lèvres légèrement moustachues, quelques gouttes dont ce n'était pas la place ${ }^{30}$.

Les éditeurs de la Recherche signalent que Saint-Simon met en scène Mme de Thiange, sœur de Mme de Montespan, dotée de la même particularité: «Elle bavait sans cesse et fort abondamment ${ }^{31}$. Mais nos lettres nous montrent que la comtesse Murat, transformant, dans son enthousiasme pour Charles Maurras, Gustave Schlumberger en hippopotame ruisselant sous les yeux amusés de Proust, constitue un modèle plus immédiat. Quant à la jeune Mme de Cambremer, née Mlle Legrandin, son snobisme est un trait de famille. Et notamment, ce que rapporte Proust de Gustave Schlumberger, gémissant du malheur de fréquenter le monde, puis courant se réfugier entre deux duchesses, rappelle à l'évidence l'attitude de Legrandin à Combray, prêchant la solitude au jeune héros, puis aperçu, au sortir de l'église, tout empressé auprès d'une châtelaine ${ }^{32}$. Seule la correspondance nous permet ici de reconstituer les éléments épars qui ont cristallisé pour former ensemble la famille Legrandin-Cambremer: le château à Cambremer, la salivation de la comtesse Murat, l'incompréhension de la musique, et la comédie de l'antisnobisme, tout cet ensemble s'organisant autour de Gustave Schlumberger.

Jean Schlumberger au contraire ne cessera de se rapprocher de Proust, au fil des ans. La pleine connivence entre les deux écrivains n'occupe, il est vrai, que les trois dernières années de la vie du romancier de la Recherche. Ce n'est en effet qu'en 1920 que Schlumberger prétend tout à coup « découvrir » Proust, lequel écrit à son ami Robert de Billy: «Cela vous amuserait-il que je vous envoie une lettre de Jean Schlumberger qui m'a récemment 'découvert'? ${ }^{33}$ Récemment, c'est-à-dire bien tard. En fait, l'auteur de Swann occupe, devant Schlumberger, la place du «nouvel écrivain» dans Le Côté de Guermantes (l'épisode paraittra d'ailleurs, dans Guermantes II, en 1921: y a-t-il eu ici influence?), et Proust médite une nouvelle fois sur le décalage entre la création et la reconnaissance d'un artiste, décalage à la source d'un perpétuel anachronisme de la critique mis en scène en ces termes: «Les gens de goût nous disent aujourd'hui que Renoir est un grand peintre du XVIII siècle. Mais en disant cela ils oublient le Temps et qu'il en a fallu beaucoup, même

${ }^{30}$ Ibidem, p. 203.

${ }^{31}$ Ibidem, p. 1457, note à ce passage.

${ }^{32}$ Idem, t. l, pp. 122-128.

${ }^{33}$ Correspondance, t. XIX, p. 673. Certaines lettres de Jean Schlumberger à Proust scmblent donc avoir été đétruites, comme beaucoup d'autres, après la mort de Proust. 
en plein XIX ${ }^{\mathrm{e}}$, pour que Renoir fût salué grand artiste» ${ }^{34},-$ en sorte qu'après avoir conspué les créateurs de l'avenir, on les fait sans transition rétrograder dans le passé.

Quoi qu'il en soit, Jean Schlumberger, contrairement à Gustave, est désormais converti à la Recherche. Il s'émerveille de lire l'extrait du Côté de Guermantes sous le titre Une agonie dans la N.R.F. du $1^{\mathrm{er}}$ janvier 1921, ainsi que Jacques Rivière en apporte aussitôt le témoignage à Proust ${ }^{35}$. Celui-ci connât d'ailleurs, depuis sa parution en 1918, une édition d'Euvres choisies, en poésie et en prose, de Walt Whitman, dont Schlumberger est l'un des traducteurs ${ }^{36}$; mais il a lu surtout, à sa parution en 1921, Un homme heureux, dont il cite au besoin des phrases précises, et qu'il confronte globalement avec la Recherche, souhaitant souligner, dit-il, «les points de contact profonds qu'Un homme heureux révèle, à mon avis, entre nous. Comme vous j'ai cherché à exprimer la joie 'quand mon cœur bondissait plus haut que les maisons', comme vous j'ai cherché à exprimer la douleur. Pourvu que vous n'ayez pas lu dans la N.R.F. 'Une agonie' [Proust sait bien que si, par Jacques Rivière] où mon idée de la mort (sur laquelle vos pages de la fin d'Un homme heureux me font revenir) est pour tant de raisons mal exprimée» ${ }^{37}$.

La relation avec Jean Schlumberger est donc d'abord de lecture, réciproque et compréhensive (même si les termes de la comparaison de Proust nous indiquent qu'il cherche à se montrer aimable plus qu'il n'est réellement admiratif), épistolaire dès lors: deux grandes lettres, écrites par l'auteur de la Recherche, livrent l'essentiel de ces échanges, le 31 mars $1921^{38}$ et le 16 juillet $1922^{39}$. Comme au temps de Gustave Schlumberger, la rencontre avec Jean pourrait se faire en société, aux «mercredis» notamment de Jacques Rivière, à partir de $1921^{40}$ ou dans la société de Pontigny, autour de Paul Desjardins ${ }^{41}$, dont Schlumberger semble un habitué; les deux confrères se trouvent même associés à des ventes de charitét ${ }^{42}$. La dernière sphère de rencontre est celle de la critique littéraire, autour de l'équipe de la N.R.F. Proust assiste à un conflit qui oppose Schlumberger à Marcel Boulenger, soup̧̧onné d'avoir opposé un veto au compte rendu d'Un homme heureux dans L'Opinion ${ }^{43}$. Jusqu'au bout, Proust restera en bons termes avec son confrère, puisqu'en 1922, c'est à lui qu'il s'adresse pour solliciter la traduction, toujours différée par l'équipe

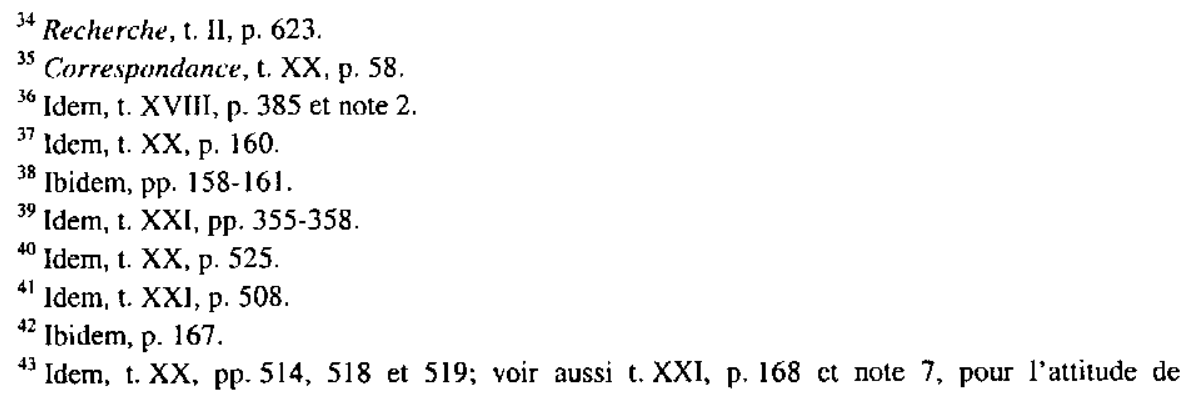

${ }^{43}$ Idem, t. XX, pp. 514, 518 et 519; voir aussi t. XXI, p. 168 et note 7, pour l'attitude de Gallimard dans ce conflit. 
de la N.R.F., d'un article d'Ernst-Robert Curtius sur la Recherche ${ }^{44}$; une lettre de mai expliquera pourquoi: «j'ai souvent reçu des pages pleines de la plus compréhensive sympathie dans un joumal de ces provinces [l'Alsace et la Lorraine], écrites par M. Jean Schlumberger» ${ }^{45}$, lequel se verra encore sollicité pour écrire un article sur la Recherche dans le «Figaro» ${ }^{46}$, tâche dont il s'acquittera.

Ce sont bien sûr ces pages pleines de la plus compréhensive sympathie qui doivent pour finir retenir notre attention: nous y trouverons des analyses qu'il est intéressant de savoir placées sous les yeux de Proust, dans la mesure où l'une d'elles a inspiré un passage célèbre du Temps retrouvé. Il s'agit en fait de deux chroniques pour l'essentiel, le Billet de la semaine paru dans L'Alsace française du 19 mars $1921^{47}$ et «À la recherche du temps perdu: une nouvelle Comédie humaine» que publiera le Figaro, dans son supplément littéraire du 16 juillet $1922^{48}$. La première des deux chroniques semble à Proust contenir des «reproches injustes», par allusion à une remarque de Schlumberger qui reprend tout ce que les journalistes avaient écrit à la parution de $D u$ côté de chez Swann, sur le prétendu manque de composition de la Recherche: «Rien ici de ce qu'on appelle communément équilibre de la composition, perfection du style, mouvement dramatique du récit. Ces mémoires, où l'autobiographie se mêle de façon inextricable à la fiction, composent déjà cinq volumes, et l'auteur n'a guère encore conduit son héros au-delà de la vingtième années ${ }^{49}$. Le romancier a si souvent répondu à ces erreurs qu'il glisse rapidement ici, d'autant qu'il reçoit aussi du critique ce.qu'il appelle des «éloges immérités»; il s'agit cette fois du style, de la phrase, analysés non sans subtilité et intuition par Schlumberger, en ces termes: «Devant les meilleures pages de Marcel Proust, on ressent les impatiences et les ravissements soudains qu'on éprouve à lire Saint-Simon ou Retz; et s'il arrive qu'on soit forcé de s'y reprendre à deux fois pour se reconnaître dans un méandre de propositions, on s'aperçoit bien vite que, sans ces détours, la phrase aurait laissé perdre le plus subtil de son contenus ${ }^{50}$. Saint-Simon constitue, y compris dans son style ${ }^{51}$, un grand modèle de Proust; par ailleurs la formulation du critique rejoint sensiblement celle par laquelle le narrateur du Côté de Guermantes décrit sa découverte du style du nouvel écrivain: «je sentais que ce n'était pas la phrase qui était mal faite, mais moi pas assez fort et agile pour aller jusqu'au bout. Je reprenais mon élan, m'aidais des pieds et des mains pour arriver à l'endroit d'où je verrais les rapports nouveaux entre les choses» ${ }^{52}$. Le nouvel écrivain, c'est au fond ici Proust face à la critique

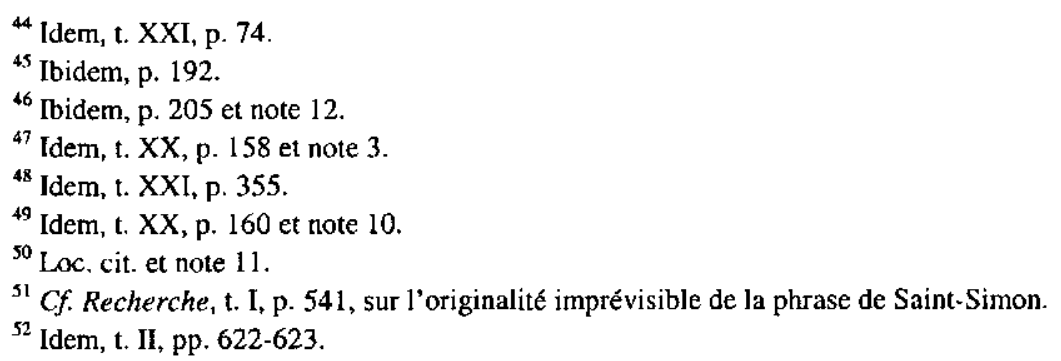


contemporaine: l'exemple de Jean Schlumberger le rappelle au romancier dogmatique.

La seconde chronique ouvre cependant un très fructueux débat. Proust n'adresse à son auteur qu'un reproche, c'est d'avoir trop mis en avant les transpositions de sexe à la source des personnages: «les transpositions de sexe que vous m'attribuez - involontairement, $\mathrm{j}$ 'en suis certain - m'ont été désagréables après tout ce qu'on a dit. Votre phrase est parfaitement logique et n'implique aucune malveillance. Mais venant après tant d'absurdes propos sur les jeunes filles hommes déguisés elle semble donner à une hypothèse insensée la consécration de votre esprit infaillible et de votre grand talent» ${ }^{53}$. Quoique faisant parfaitement la part des choses, et reconnaissant pleinement les intentions bienveillantes de Schlumberger, Proust se montre ici, il est plaisant de le noter pour finir, doublement de mauvaise foi. C'est pour préserver sa personnalité sociale que le romancier rejette comme une absurdité toute idée de transposition, car dans l'intimité d'autres lettres, il l'admet volontiers. Il est vrai que nous en conservons de rares témoignages, dont ce message à Reynaldo Hahn, à qui l'auteur de Swann vient de confier, en octobre 1914, son chagrin d'avoir perdu Agostinelli: «Prière de ne parler de tout cela à personne; si le caractère général de ces vérités vous donnait la tentation d'en lire quelques extraits à Gregh ou à d'autres, vous me feriez beaucoup de peine. Si jamais je veux formuler de telles choses, ce sera sous le pseudonyme de Swann ${ }^{54}$.

Mais le plaisant est que, précisément, Proust va dès lors reprendre la phrase incriminée de Schlumberger, la transposer, non d'un personnage à l'autre ou d'un homme à une femme, mais de ce thème à la création même des personnages, et en faire une phrase à ce sujet célèbre du Temps retrouvé. Car au fond, qu'avait écrit le critique? «Il est bien vrai qu'on reconnaît parfois une figure, un caractère, une anecdote; mais l'artiste a toujours usé très librement de ses observations, prêtant à l'un les aventures de l'autre, plaçant une tête sur des épaules qui ne lui appartenaient pas, faisant d'un adolescent une jeune fille ou d'une douairière un vieux monsieur ${ }^{55}$. L'image n'est pas perdue pour le romancier, dont la main aussitôt atteint les cahiers consacrés au chapitre de «L'Adoration perpétuelle» qui dérouleront tous ses principes sur la création littéraire; il y inscrit, ou du moins y retouche alors ces morceaux de phrase, sur le romancier créateur de personnages: «quand il écrit, il n'est pas un geste de ses personnages, un tic, un accent, qui n'ait été apporté à son inspiration par sa mémoire, il n'est pas un nom de personnage inventé sous lequel il ne puisse mettre soixante noms de personnages vus, dont l'un a posé pour la grimace, l'autre pour le monocle, tel pour la colère, tel pour le mouvement avantageux du bras», - «emmanchant sur ses épaules un mouvement de cou fait pas un autre, chacun ayant donné son instant de pose» ${ }^{56}$. Gustave et Jean Schlumberger,

\footnotetext{
${ }^{53}$ Correspondance, t. XXI, p. 356.

${ }^{\$ 4}$ Idem, t. XIV, p. 358.

55 Idem, t. XXI, p. 357, note 6; nous soulignons.

${ }^{56}$ Recherche, t. IV, pp. 478-479; nous soulignons.
} 
l'un en société, l'autre dans ses écrits, ne forment-ils pas, fondus ensemble, cet artiste à double personnalité que Proust oppose à Sainte-Beuve? À la faveur de ces répartitions et de ces échanges, le romancier a puisé dans la mondanité littéraire pour méditer in extremis - quelques mois avant sa mort, et dans les tout derniers cahiers de la Recherche - sur les mystères de la chimie créatrice. 